\title{
Gut Microbiome and Human Health
}

\author{
Heba N.Gad EL-Hak ${ }^{a *}$, Abdel Raouf A. Moustafa ${ }^{\mathrm{b}}$ and Samira R. Mansour \\ Department of Zoology, Egypt
}

*Corresponding author: Heba Nageh Gad EL Hak, Department of Zoology, Egypt

\begin{abstract}
The human gut microbiota is given as an especially complicated microbial community that are formed to possess a significant impact on human physiology. Additionally, comparative analysis of individual human gut microbiota has discovered numerous methods that the microbiota use to regulate to the enteric surroundings. Infections of the alimentary canal are a significant pathological state for each adults and children worldwide. Alterations within the traditional human gut microflora lead to the event of enteric upsets. Infective bacterium alters the enteric biology and enteric organization resistance. A healthy gastrointestinal microbiota forms a barrier against invasive organisms. Traditional enteric microbes and a few probiotic bacterium will enhance the host's defense mechanisms against pathogens. They'll additionally improve enteric immunity by adhering to the enteric tissue layer and stimulating native immune responses. The upkeep of a balanced enteric biology improves the ability to preserve enteric integrity. The cancer patient microbiota is completely different from healthy one, conjointly the chemotherapy received by the cancer patient have an effect on the microbiota and may cause different sickness.
\end{abstract}

Keywords: Gut Microbiota; Cancer patient microbiota; Rat microbiota

\section{Introduction}

A large diversity of microorganisms be within the mammalian gastrointestinal tract, with their variety around ten times bigger than the whole number of mammalian somatic and germ cells [1] these sizable amounts ensuing from the mammalian completely different niches with distinct physicochemical conditions [2]. The microbiota inhabiting the GI tract constitutes a fancy scheme and plays a important role in maintaining host physiological physiological state [3]. A large body of analysis has investigated the gut flora composition in humans [4] and disclosed its relationship to diseases [5]. However, most of the samples utilized in these research were from faecal matter [6] or primarily through hospitalbased endoscopic biopsies [7]. Major functions of the gut microflora include metabolic activities that lead to salvage of energy and absorbed nutrients, necessary biological process effects on intestinal epithelia and on immune structure and performance, and protection of the colonized host against invasion by alien microbes. Gut flora may also be an important factor in certain pathological disorders, as well as multisystem organ failure, carcinoma, and inflammatory intestinal diseases. Even so, bacteria also are helpful in promotion of human health. Probiotics and prebiotics are best- known to possess a role in the prevention or treatment of some diseases [5]. Due to limitations in human research, murine models as rat became crucial in studies of the gut microbiota designed to get mechanistic insights into its completely different anatomical regions. As a long-standing model in biomedical analysis, rats have recently been utilized in various studies exploring the correlations between intestinal microorganism biota and numerous types of diseases with the comparative characterization of the traditional rat microbiota landscape [8-10]. The rat's large intestine houses a lot of complicated micro-ecosystem, that was additionally supported by earlier research indicating that the amount of microorganism species of rat fecal matter was 2-3 times over that of human feces at the identical sequencing effort [11]. Lactobacillus, a typical commensal organism, sometimes highly abundant within the laboratory rodent gut, it's predominate within the stomach and higher part of the small intestine and related to keratinized cells of the nonglandular portion of the stomach that controlled the population levels of different bacterial species [12]. Within the lower part of the small intestine, another lactateproducing bacteria within the upper GI tract most likely explains 
the enrichment of lactate within the stomach and small intestine, anaerobic Turicibacter, was additionally predominant within the rat GI tract given the oxygen availability within the upper GI tract. The saccharolytic bacterium residing within the outer mucus layer might digest mucin glycans [13] and facilitate build polypeptides additional accessible for proteolytic bacterium, enhancing syntrophic interactions. Staphylococci isolated from a conventional rat colonized specifically the keratinized cells of the nonsecreting epithelial tissue of the stomach when the rats were free from lactobacilli. This colonization wasn't discovered when inoculation of into the rats [14].

\section{Chemotherapy and microbiota}

The gut microbiota of the cancer patient is affected that confirmed by the study of [15]. The intestinal microbiota clostridium leptum and C. coccoid is habitually cited as a possible aetiological consider colorectal cancer initiation and progression and are considerably altered in both colorectal cancer and polypectomized subjects compared with controls [15]. Additionally, throughout chemotherapy treatment the gut microbiota is affected that defend the body from the infection which confirmed by the study of [16] analyzed the impact of thirty six chemotherapy cycles treatment with an antimicrobial prophylaxis on intestinal microorganism populations (microbiota) among pediatric patients with acute myeloid leukemia who are susceptible to intestinal mucositis and infections. Throughout thirty six therapy cycles of daunorubicin, etoposide, and cytarabine to see whether or not the decrease within the numbers of bacterium was a result of therapy, the sensitivity of various bacterial species to chemotherapeutic used. In vitro, each daunorubicine and etoposide showed a negative result on the expansion of each anaerobic and aerobic bacterium whereas using a higher concentration of etoposide, however not daunorubicine resulted during a more decrease in bacterial growth. In distinction, cytarabine showed no result in the expansion of the tested bacterial strains. They all over that patients with acute myeloid leukemia treated with therapy and prophylactic antibiotics are unable to keep up colonization resistance and disturb the balance between anaerobic and aerobic bacterium as a result of that a decrease within the anaerobic bacterium and a rise in potentially the danger of infective aerobic enterococci infections among immunocompromised patients with cancer. Some therapy worked by stimulating the antitumor immune reaction which will alter the gut microbiota because the Cyclophosphamide alters the composition of microbiota within the small intestine and induces the translocation of selected species of gram-positive bacterium into secondary lymphoid organs. There, these bacterium stimulate the generation of a particular subset of "pathogenic" $\mathrm{T}$ helper 17 (pTH17) cells and memory TH1 immune responses [17]. The foremost of therapy, medication elicited diarrhea which will cause intestinal alterations which can lead to diminished bacterial functions within the gut, altering gut function and initiating intestinal damage [18] with quantitative real-time PCR showed that patients with diarrhea exhibit variations in fecal microbiota bacterial composition compared with healthy controls, with a trend towards a decrease in lactobacillus spp., bacteroides spp., Bifidobacterium spp., Enterococcus spp. and a trend towards a rise in Escherichia coli and staphylococci spp. Cancer patients with diarrhea additionally exhibit a trend towards decreasing methanogenic archaea compared to healthy people [19] found in stool samples and blood samples at numerous times throughout the therapy cycle of Twenty-six cancer patients showed decreases in lactobacillus spp., Bifidobacterium spp., bacteroides spp. and Enterococcus spp. and an increase determined in E. coli and cocci spp. Methanogenic archaea were additionally quantified, with all patients except one showing a decrease. Fecal calprotectin levels were accumulated, circulating MMP-3 and MMP-9 significantly increased following therapy. Whereas the circulating levels of NF$\kappa B, I L-1 \beta$ and tumor necrosis factor was nonsignificant increased following therapy.

\section{Conclusion}

The gut microbiota consists of many trillion of bacterial cells, that exceed the amount of somatic and germ cells present within the human body by a factor of ten. These bacteria serve as a barrier against pathogens and are crucial for the development of the host immune system. Different interesting data that may be obtained through this study strategy consideration the impact of chemotherapy on the human gut microbiota. Finally, it'll become potential to identify microbiota, which will be used as biomarkers for health, for condition of sickness and for treatment. Finally, the identification of the gut microbiota in patients are going to be important so as to elucidate treatment of disease.

\section{References}

1. Turroni F, Ribbera A, Foroni E, Sinderen VD, Ventura M (2008) Human gut microbiota and bifidobacteria: From composition to functionality. Antonie Van Leeuwenhoek 94(1): 35-50.

2. Dethlefsen L, Eckburg PB, Bik EM, Relman DA (2006) Assembly of the human intestinal microbiota. Trends in Ecol Evol 21(9): 517-523.

3. Hooper LV, Macpherson AJ (2010) Immune adaptations that maintain homeostasis with the intestinal microbiota. Nat Rev Immunol 10(3): 159-169.

4. Eckburg PB, Bik EM, Bernstein CN, Purdom E, Dethlefsen L, et al. (2005) Diversity of the human intestinal microbial flora. Science 308(5728): 1635-1638.

5. Guarner F, Malagelada JR (2003) Gut flora in health and disease. Lancet 361(9356): 512-519.

6. Franks AH, Harmsen HJ, Raangs GC, Jansen GJ, Schut F, et al. (1998) Variations of bacterial populations in human feces measured by fluorescent in situ hybridization with group-specific 16s rRNA-Targeted oligonucleotide probes. Applied and environmental microbiology 64: 3336-3345.

7. Dicksved J, Lindberg M, Rosenquist M, Enroth H, Jansson JK, et al. (2009) Molecular characterization of the stomach microbiota in patients with gastric cancer and in controls. J Med Microbiol 58(4): 509-516.

8. Islam KS, Fukiya S, Hagio M, Fujii N, Ishizuka S, et al. (2011) Bile acid is a host factor that regulates the composition of the cecal microbiota in rats. Gastroenterology 141(5): 1773-1781. 
9. Collins SM, Bercik P (2009) The relationship between intestinal microbiota and the central nervous system in normal gastrointestinal function and disease. Gastroenterology 136(6): 2003-2014.

10. Round JL, Mazmanian SK (2009) The gut microbiota shapes intestinal immune responses during health and disease. Nat Review Immunol 9(5): 313-323.

11. Campbell JM, Fahey GC, Wolf BW (1997) Selected indigestible oligosaccharides affect large bowel mass, cecal and fecal short-chain fatty acids, PH and microflora in rats. J Nutr 127(1): 130-136.

12. Umesaki Y, Setoyama H (2000) Structure of the intestinal flora responsible for development of the gut immune systemin a rodent model. Microbes Infect 2(11): 1343-1351.

13. Li D, Chen H, Mao B, Yang Q, Zhao J, et al. (2017) Microbial biogeography and core microbiota of the rat digestive tract. Sci Rep 8.

14. Sekirov I, Russell SL, Antunes ICM, Finlay BB (2010) Gut microbiota in health and disease. Physiol Rev 90(3): 859-904.
15. Scanlan PD, Shanahan F, Clune Y, Collins JK, O Sullivan GC, et al. (2008) Culture-independent analysis of the gut microbiota in colorectal cancer and polyposis. Environ Microbiol 10(3): 789-798.

16. Van Vliet MJ, Tissing WJ, Dun CA, Meessen NE, Kamps WA, et al. (2009) Chemotherapy treatment in pediatric patients with acute myeloid leukemia receiving antimicrobial prophylaxis leads to a relative increase of colonization with potentially pathogenic bacteria in the gut. Clin Infect Dis 49(2): 262-270.

17. Viaud S, Saccheri F, Mignot G, Yamazaki T, Daillère R, et al. (2013) The intestinal microbiota modulates the anticancer immune effects of cyclophosphamide. Science 342(6161): 971-976.

18. Stringer AM, Al Dasooqi N, Bowen JM, Tan TH, Radzuan M, et al. (2013) Biomarkers of chemotherapy-induced diarrhoea: A clinical study of intestinal microbiome alterations, inflammation and circulating matrix metalloproteinases. Support Care Cancer 21(7): 1843-1852.

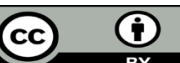

This work is licensed under Creative Commons Attribution 4.0 License

To Submit Your Article Click Here:

Submit Article

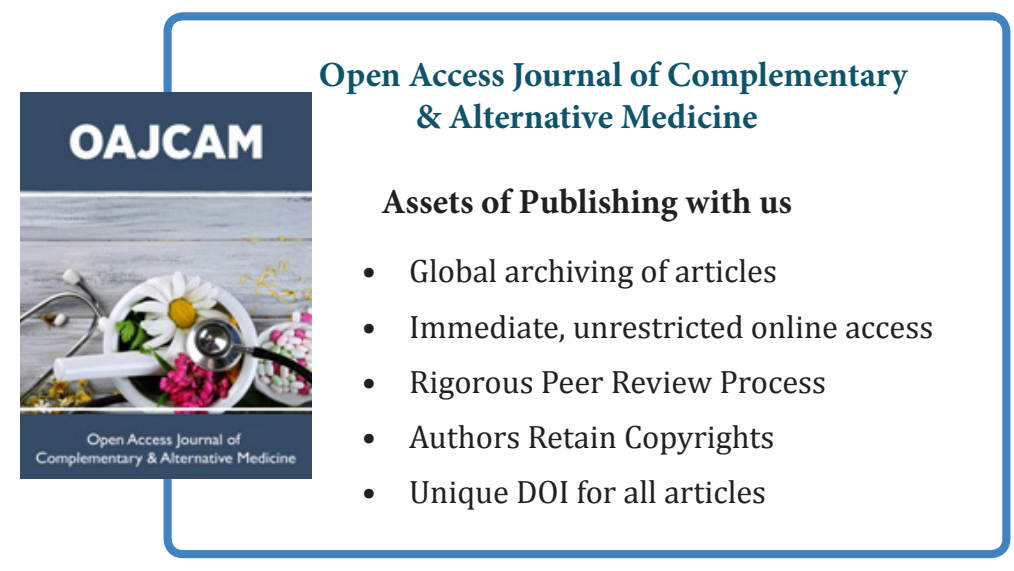

